\title{
Importância e aplicabilidade da Norma ABNT NBR 5410/2004 no quesito verificação final: segurança e confiabilidade do serviço
}

Este trabalho tem como tema 'Importância e aplicabilidade da norma ABNT NBR 5410/2004 no quesito verificação final: segurança e confiabilidade do serviço'. A NBR 5410/2004 normatiza as instalações elétricas de baixa tensão e expõe que a verificação final leva à certificação da rede de energia elétrica, quando não apresentam danos aparentes que possam comprometer seu funcionamento adequado e a segurança. Desta maneira este trabalho tem como objetivo confirmar por meio de revisões de literatura, a importância da avaliação de integridade das instalações elétricas, conforme preconiza a norma NBR 5410/2004, e posteriormente sugerir um checklist a ser utilizado na verificação final. Os resultados da pesquisa demonstraram que a importância da avaliação da conformidade das instalações elétricas, após as leituras, verificou-se que a mesma está pautada na segurança dos usuários, redução de retrabalho, benefícios para a concessão e na vida útil das instalações. Por fim, concluiu-se que ao finalizar uma obra e/ou durante a sua execução é importantíssimo que se realize a inspeção de certificação da instalação elétrica, sendo que a mesma é constituída por vistoria documental e visual.

Palavras-chave: NBR 5410; Verificação Final; Instalações Elétricas; Conformidades.

\section{Importance and applicability of Standard ABNT NBR 5410/2004 in the question final verification: safety and reliability of the servisse}

This work has as its theme 'Importance and applicability of the ABNT NBR 5410/2004 standard in the final verification: security and reliability of the service'. The NBR 5410/2004 regulates the low voltage electrical installations and exposes that the final verification leads to the certification of the electric power network when they do not present apparent damages that may compromise its proper functioning and safety. In this way, this work aims to confirm, through literature reviews, the importance of the evaluation of the integrity of the electrical installations, as recommended by the standard NBR 5410/2004, and later to suggest a checklist to be used in the final verification. The results of the research demonstrated that the importance of evaluating the conformity of electrical installations after the readings has been verified that it is based on user safety, reduction of rework, benefits for the concession and on the life of the installations. Finally, it was concluded that at the end of a work and / or during its execution, it is extremely important to carry out the inspection of the electrical installation certification, which is constituted by a documentary and visual inspection.

Keywords: NBR 5410; Final Verification; Electrical Installations; Conformities.

\section{Topic: Engenharia Elétrica}

Reviewed anonymously in the process of blind peer.
Received: 09/01/2019

Approved: 11/04/2019

Kaike Eduardo Neves Lima

Instituto Tocantinense Presidente Antônio Carlos, Brasil

http://lattes.cnpq.br/6062574343710619

kaikelima16@gmail.com

Eduardo Gouveia Santiago Lage

Instituto Tocantinense Presidente Antônio Carlos, Brasil

http://lattes.cnpq.br/4344949197847151

eduardogslage@gmail.com

Referencing this:

LIMA, K. E. N.; LAGE, E. G. S.. Importância e aplicabilidade da Norma ABNT NBR 5410/2004 no quesito verificação final: segurança e confiabilidade do serviço. Technology Science, v.1, n.1, p.1-6, 2019 DOI: http://doi.org/10.6008/CBPC2674-6425.2019.001.0001 


\section{INTRODUÇÃO}

A partir do ponto de vista da utilização final de energia elétrica, as instalações elétricas de baixa tensão representam o meio de ligação entre a fonte fornecedora e os equipamentos finais. Para garantir a segurança dos usuários e a eficiência dos processos envolvidos, não basta apenas possuir um projeto correto e/ou utilizar produtos tecnicamente conforme, se o serviço da instalação não for executado por pessoas qualificadas, que utilizem corretamente as técnicas necessárias para uma boa instalação elétrica. É importante destacar que, caso isso não aconteça, pode-se comprometer a segurança dos usuários, bem como os critérios operacionais da instalação e sua eficiência (DANIEL, 2013).

Uma instalação elétrica adequada e o uso correto dos equipamentos são as exigências necessárias para usufruir a energia elétrica em uma edificação, sendo que estas condições estão preconizadas pela norma técnica brasileira NBR 5410/2004, à qual vem sugerindo as instalações elétricas de baixa tensão, tanto residenciais quanto comerciais e industriais, além de ter como objetivo, em estabelecer, as condições das instalações elétricas de baixa tensão, com o intuito de garantir a segurança de pessoas e animais, o funcionamento adequado e a conservações dos bens (ABNT, 2004).

No capítulo 7 da NBR 5410/2004 é apresentado os procedimentos necessários para se fazer uma verificação final das instalações elétricas, antes de serem colocadas em uso, sendo que está verificação tem a finalidade de garantir que tudo o que foi executado estará de acordo com as exigências e prescrições contidas na norma (LIMA JUNIOR, 2013).

Tamietti (2002) acrescenta que a verificação das instalações elétricas devem ser feitas antes do seu funcionamento, onde a verificação deve ser realizada em todo o tipo de construção, independente se é residencial, comercial ou industrial, seguindo sempre as características de cada instalação.

A verificação, segundo Martinho (2015), é uma ação que consiste em "um conjunto de procedimentos, que devem ser definidos antes do início da obra, visto que determinadas verificações podem ser realizadas durante a execução da instalação". Então, a verificação poderá ser realizada no final da obra ou no seu decorrer, ressaltando que a mesma deve ser feita antes do funcionamento.

Segundo a NBR 5410/2004, item 7.1.5, a verificação só pode ser realizada por profissionais qualificados, com experiência e competência em inspeções, ou seja, somente profissionais com curso devidamente reconhecido pelos órgãos competentes, poderão realizar uma inspeção e emitir, formalmente, relatórios e laudos avaliando determinada instalação elétrica (ABNT, 2004).

Existem países que já exigem a certificação da conformidade das instalações elétricas antes da sua entrega, porém, mesmo que nestes países tenha verificado a utilização de produtos certificados, os quais possuem o selo de qualidade excelente, não garantem a excelência na instalação elétrica (LIMA JUNIOR, 2013).

No Brasil, essa condição ainda não é uma realidade, porém possui requisitos técnicos que devem ser aplicados ao se realizar uma instalação elétrica de baixa tensão, embora a solução para minimizar e até mesmo eliminar os problemas seria uma inspeção exigida por um órgão máximo, pois, desta maneira, seria 
possível garantir a segurança da sociedade e a seriedade dos profissionais diretamente envolvidos (RIBEIRO, 2016). Enquanto, essa obrigatoriedade legal não acontece, é importante destacar que nada impede que esta avaliação de conformidades seja realizada, sendo que para tanto é indispensável que se cumpra a NBR $5410 / 2004$.

Com base no exposto, uma problemática se faz presente nesta proposta de pesquisa, sendo este: 'Qual a importância da avaliação das conformidades das instalações elétricas, conforme preconizado pela NBR 5410/2004?'. Portanto, o objetivo desta pesquisa foi confirmar, por meio de revisões de literatura, a importância da avaliação da conformidade das instalações elétricas, conforme é preconizado pela NBR 5410/2004 e, posteriormente, sugerir um checklist a ser utilizado na verificação final.

\section{METODOLOGIA}

Trata-se de um estudo secundário, uma vez que buscou estabelecer conclusões a partir de estudos primários, que já foram publicados na literatura, realizando uma revisão bibliográfica do tipo descritiva com abordagem qualitativa, pois, a pesquisa do tipo descritiva, observa, registra e analisa fenômenos, sem manipulá-los, procurando descobrir a frequência, sua natureza, características e sua relação com outros fenômenos. A abordagem qualitativa se preocupa, com as ciências sociais, com um nível de realidade que não pode ser quantificado, apenas analisado e estudado.

Sendo elaborado, após estudo das revisões bibliográficas, bem como do estudo e análise da NBR 5410/2004, um checklist que poderá ser utilizado na verificação final da instalação elétrica. A amostra foi composta por artigos, monografias, normas e periódicos publicados em revistas nacionais com data de publicação compreendida entre os últimos seis anos. A base de dados para levantamento dos artigos foram o Google Acadêmico, a Biblioteca da Escola de Engenharia UFRGS - BIBENG e outros.

\section{RESULTADOS}

Os resultados apresentados partiram da revisão de literatura, pois a base de dados da pesquisa realizada foram a seleção de dezoito publicações, distribuídas entre artigos, monografias e periódicos, porém, durante a análise das publicações, nove foram excluídas, permanecendo nove publicações na base. As exclusões foram feitas devido as produções possuírem datas de publicação superior a sete anos, bem como por não terem como foco principal as instalações elétricas de baixa tensão, assim as publicações selecionadas e utilizadas estão descritas na Tabela 1.

As publicações selecionadas para o desenvolvimento desta pesquisa são considerados recentes, uma vez que possuem ano de publicação de 2016 a 2018, portanto as informações que aqui serão disponibilizadas são consideradas atuais. Quanto a importância da avaliação da conformidade das instalações elétricas, após as leituras, verificou-se que a mesma está pautada em:

1) Segurança dos usuários, sendo este a principal vantagem de uma inspeção, uma vez que irá reduzir riscos contra os principais causadores de acidentes, como o incêndio gerado por curtos-circuitos e o temível choque elétrico; 
2) Redução de retrabalho, uma vez que, por meio da inspeção de certificação, é possível realizar o levantamento das não-conformidades e, tão logo estes, sejam identificadas, para enfim realizar os ajustes necessários. Quando a verificação é realizada dentro do processo de inspeção, o custo de retrabalho é reduzida, uma vez que a obra ainda está em fase de testes e não foi colocada em uso;

3) Benefícios para a concessão, pois, ao certificar que as instalações elétricas cumprem o que está estabelecido na NBR 5410, ocorre uma diminuição das perdas técnicas, trazendo um melhor aproveitamento da energia;

4) Vida útil das instalações, uma vez que, por meio da inspeção é possível verificar se ocorreu o adequado dimensionamento dos componentes da instalação, evitando operações de componentes incorretos durante as operações de funcionamento. Assim a inspeção garante uma vida útil das peças, aproveitando-os ao máximo, sem falar na diminuição dos custos de manutenção e de novos investimentos a longo prazo.

Tabela 1: Demonstração das publicações selecionadas segundo o título, autores, ano de publicação.

\begin{tabular}{|c|c|c|}
\hline TítULO & AUTOR & $\begin{array}{c}\text { ANO DE } \\
\text { PUBLICAÇÃo }\end{array}$ \\
\hline $\begin{array}{c}\text { Desenvolvimento de aplicativo computacional para cálculo de demanda segundo as } \\
\text { normas de distribuição da CEB e dimensionamento de condutores e dispositivos de } \\
\text { proteção segundo a ABNT NBR 5410:2004 }\end{array}$ & $\begin{array}{c}\text { NEVES JUNIOR, } \\
\text { Fernando José das. }\end{array}$ & 2014 \\
\hline $\begin{array}{c}\text { Inspeção das Instalações Elétricas do bloco K, L e M do Centro de Tecnologia da } \\
\text { Universidade Federal da Paraíba }\end{array}$ & $\begin{array}{c}\text { SANTANA, Wolney } \\
\text { Lucena }\end{array}$ & 2016 \\
\hline Implantação do prontuário de instalações elétricas (PIE) & BRAIDA, Valéria & 2016 \\
\hline $\begin{array}{c}\text { A gestão eficiente de projetos de instalações elétricas para edificações } \\
\text { PEREIRA, Jose } \\
\text { Henrique Alves }\end{array}$ & 2016 \\
\hline $\begin{array}{c}\text { BRONZATO, Max } \\
\text { Alberto }\end{array}$ & 2016 \\
\hline Projeto de instalações elétricas em baixa tensão & $\begin{array}{c}\text { CÂMARA, Andrew } \\
\text { Augusto Santos }\end{array}$ & 2017 \\
\hline A importância da conformidade das instalações elétricas para a gestão de riscos e \\
prevenção de incêndios em patrimônio cultural edificado & $\begin{array}{c}\text { SILVINO, Marcelo } \\
\text { Santana }\end{array}$ & 2018 \\
\hline Revisão da norma ABNT NBR 5410: 2004 instalações elétricas de baixa tensão & $\begin{array}{c}\text { LIMA, Letícia } \\
\text { Stephanes }\end{array}$ & 2018 \\
\hline Avaliação de conformidade das instalações elétricas de baixa tensão: sua & $\begin{array}{c}\text { SUMARIVA, } \\
\text { Ezequiel; SILVA, } \\
\text { Fabiano }\end{array}$ & 2018 \\
\hline
\end{tabular}

\section{DISCUSSÃO}

Sobre a certificação, verificou-se que a mesma é primordial para o bom funcionamento das instalações elétricas, embora, no Brasil, não exista uma lei que exija que a certificação seja realizada nas edificações, porém ressalta-se que a mesma é uma ação necessária para a prevenção de vários problemas.

Em um trabalho realizada por Silvino (2018), o qual teve como objetivo colaborar para a gestão do patrimônio cultural edificado, apresentou diretrizes de intervenções para adequações das instalações elétricas. Na pesquisa, o autor afirma que a eletricidade é o segundo maior fator de ativação de incêndio em residências e que o mesmo não ocorre por falhas na eletricidade e sim pela ignição do material isolante dos condutores ou do quadro de distribuição de energia, sendo que quando é realizada a inspeção de certificação das instalações, esses riscos são diminuídos.

É importante destacar que o Brasil possui a Portaria no 51/2014 que rege sobre a certificação das instalações de baixa tensão, porém a mesma ainda é voluntária devido o Brasil não ter definido um órgão regulamentador para as instalações elétricas. 
A este respeito, Bronzato (2016) acrescenta que, ainda que existam normas a respeito das instalações elétricas em nosso país, estando no mesmo nível das normas internacionais, as mesmas, na prática, pouco contribuem para a melhoria da qualidade das instalações elétricas, uma vez que não existe obrigatoriedade de aplicação, gerando uma grande desigualdade entre o que as normas indicam e o que realmente é realizado nas instalações elétricas por todo o país.

A NBR 5410/2004 recomenda que após a finalização da execução da obra é importante realizar a inspeção visual e ensaio nas obras durante e/ou quando concluída a instalação, antes de ser colocada em serviço pelo usuário final, sendo que esta inspeção é necessária para a verificação da conformidade com as prescrições da norma (PEREIRA, 2016).

É importante destacar que o principal objetivo da NBR 5410, Norma de instalações elétricas de baixa tensão, tem, de forma geral, estabelecer as condições que devem satisfazer as instalações elétricas de baixa tensão, objetivando garantir a segurança das pessoas e dos animais, além de seu funcionamento adequado (CÂMARA, 2017).

Segundo Lima (2018) é por meio das normas e de uma rígida avaliação de conformidade que se consegue afirmar a qualidade nas instalações elétricas, sendo que ao realizar a avaliação e/ou inspeção das conformidades é possível entregar à população uma energia limpa e estável, de maneira econômica e principalmente segura.

A inspeção das conformidades é realizada por meio da inspeção visual, assim é possível verificar e inspecionar a execução das instalações, certificando-se de que os componentes foram selecionados e instalados corretamente, além de averiguar se eles não apresentam danos que possam comprometer o seu funcionamento e/ou a segurança dos usuários. A Portaria 51/2014 do INMETRO, descreve algumas etapas a serem seguidas para a realização da inspeção das conformidades, sendo estes: análise documental, inspeção visual e ensaios (SUMARIVA et al., 2018).

Santana (2016) realizou uma pesquisa que teve como objetivo inspecionar as instalações elétricas dos blocos K, L e M do Centro de Tecnologia da Universidade Federal da Paraíba e constataram que, com base nas inspeções realizadas, identificou-se itens não conformes com a norma, o que demonstra a importância da certificação das instalações elétricas.

\section{CONCLUSÃO}

Assim, a partir da pesquisa realizada neste trabalho, pode-se identificar que uma instalação elétrica inicia-se com a criação do projeto, sendo que para se conseguir uma boa qualidade e bons resultados, é necessário que as normas regulamentares sejam respeitadas, em especial a NBR 5410/2004. Pois, final de uma obra e/ou durante a sua execução é importantíssimo que se realize a inspeção de certificação da instalação elétrica, sendo que a mesma é constituída por vistoria documental e visual. Verificou-se, ainda, que a mesma não é obrigatória no Brasil, porém possui um papel fundamental para supervisionar todo os processos, trazendo uma série de benefícios para os envolvidos. 
Ao se analisar a importância da avaliação de conformidade das instalações elétricas, conforme preconizado pela NBR 5410/2004, verificou-se que a mesma está pautada em garantir segurança dos usuários, reduzir o retrabalho, trazer benefícios para a concessionária e preservar pela vida útil das instalações elétricas. Finalizando, neste trabalho, a sugestão de um checklist que poderá ser utilizado na verificação final da instalação elétrica, sendo que o mesmo estará apresentado em seus anexos.

\section{REFERÊNCIAS}

ABNT. NBR 5410:2004: Instalações elétricas de baixa tensão. 2 ed. Rio de Janeiro: ABNT, 2004

BRAIDA, V.. Implantação do prontuário de instalações elétricas. Monografia (Bacharelado em Engenharia Elétrica) Universidade Regional do Noroeste do Estado do Rio Grande do Sul, ljuí, 2016.

BRONZATO, M. A.. Introdução da certificação das instalações elétricas em obras públicas. Monografia (PósGraduação em Gestão de Sistemas de Engenharia) Universidade Católica de Petrópolis, Petrópolis, 2016.

CÂMARA, A. A. S.. Projeto de instalações elétricas em baixa tensão. Projeto Técnico (Bacharelado em Engenharia) Universidade Federal do Rio Grande do Norte, Natal, 2017.

DANIEL, E.. A segurança e eficiência energética nas instalações elétricas prediais: um modelo de avaliação. Monografia (Especialização) - Universidade de São Paulo, São Paulo, 2013.

LIMA, L. S.. Revisão da Norma ABNT NBR 5410:2004: Instalações Elétricas de Baixa Tensão. Projeto Técnico (Bacharelado em Engenharia Elétrica) - Universidade Federal do Rio de Janeiro, Rio de Janeiro, 2018.

LIMA JUNIOR, L. C. M.. Certificação em instalações elétricas de baixa tensão, importância e aplicabilidade da norma ABNT NBR 5410/2004. Monografia (Graduação) Universidade Federal do Paraná, Curitiba, 2013.

MARTINHO, E.. Introdução: verificação das instalações elétricas, Parte 1. 2015.

NEVES JUNIOR, F. J.. Desenvolvimento de aplicativo computacional para cálculo de demanda segundo as normas de distribuição da CEB e dimensionamento de condutores e dispositivos de proteção segundo a ABNT NBR 5410:2004. Projeto Técnico (Bacharelado em Engenharia Elétrica) - Universidade de Brasília, Brasília 2014.

PEREIRA, J. H. A.. A gestão eficiente de projetos de instalações elétricas para edificações. Monografia (PósGraduação em Gestão de Sistemas de Engenharia) Universidade Católica de Petrópolis, Petrópolis 2016.

RIBEIRO, A. E.. Readequação da planta elétrica das salas destinadas aos laboratórios de eletrônica, automação e circuitos elétricos, do Instituto Federal de Minas Gerais/ Campus Formiga. Monografia (Graduação) - Instituto Federal de Minas Gerais, Belo Horizonte, 2016.

SANTANA, W. L.. Inspeção das Instalações elétricas do bloco K, L e M do Centro de Tecnologia da Universidade Federal da Paraíba. Monografia (Bacharelado em Engenharia Elétrica) - Universidade Federal da Paraíba, João Pessoa, 2016.

SILVINO, M. S.. A importância da conformidade das instalações elétricas para a gestão de riscos e prevenção de incêndios em patrimônio cultural edificado. Monografia (Pós-graduação em Ambiente Construído e Patrimônio Sustentável) - Universidade Federal de Minas Gerais, Belo Horizonte, 2018.

SUMARIVA, E.; SILVA, F.. Avaliação de conformidade das instalações elétricas de baixa tensão: sua importância, seu processo de realização e suas vantagens. Monografia (Bacharelado em Curso de Engenharia Elétrica e Telemática) - Universidade do Sul de Santa Catarina, Curitiba, 2018.

TAMIETTI, R. P.. Avaliação da conformidade das instalações elétricas: verificação, inspeção e ensaios. Brasília: Vert Engenharia, 2002

A CBPC - Companhia Brasileira de Produção Científica (CNPJ: 11.221.422/0001-03) detém os direitos materiais desta publicação. Os direitos referem-se à publicação do trabalho em qualquer parte do mundo, incluindo os direitos às renovações, expansões e disseminações da contribuição, bem como outros direitos subsidiários. Todos os trabalhos publicados eletronicamente poderão posteriormente ser publicados em coletâneas impressas sob coordenação da Sapientiae Publishing, da Companhia Brasileira de Produção Científica e seus parceiros autorizados. Os (as) autores (as) preservam os direitos autorais, mas não têm permissão para a publicação da contribuição em outro meio, impresso ou digital, em português ou em tradução. 